\title{
BARRERAS QUE DIFICULTAN LA PLANEACIÓN ESTRATÉGICA EN LAS ORGANIZACIONES
}

\author{
BARRIERS THAT DIFFICULT STRATEGIC PLANNING IN \\ ORGANIZATIONS
}

\section{BARREIRAS QUE DIFICULAM O PLANEJAMENTO ESTRATÉGICO NAS ORGANIZAÇÕES}

\section{FERNÁNDEZ HURTADO_Saúl Rick, MARTÍNEZ MARTÍNEZ _Luz Ángela, NGONO FOUDA_ Regine Adele}

Postdoctor en Globalización y Desarrollo Regional, Center for Global Studies, Shanghai University. PhD., en Ciencias de la Administración e Ingeniería, Shanghai University. Email: saulrick@163.com, Colombia.

Profesional en Ingeniería Industrial, Departamento de Operaciones y Sistemas, Universidad Autónoma de Occidente, Email: angelamtz5th@hotmail.com, Colombia.

Ph.D., in Logistic Management and Engineering, Shanghai Maritime University - China. Master in international trade, East China University of Science \& Technology, China. Email: reginemilena@yahoo.fr, China.

Recibido: 4 de marzo del 2019

Aprobación definitiva: 14 de julio de 2019

DOI: http://dx.doi.org/10.22267/rtend.192001.108

\section{RESUMEN}

En la actualidad muchas pequeñas organizaciones en el siglo XXI fracasan debido a la falta de implementación de una planeación estratégica y a la inexistencia de un plan de negocios que les permita trazar las vías para alcanzar cada uno de sus objetivos y metas. Esta situación resulta bastante preocupante ya que, debido a esto son muchas las organizaciones que cesan sus actividades y 
terminan yéndose a la quiebra perjudicando la economía y frenando el desarrollo nacional en el escenario actual (Siglo XXI). Es por esta razón que en este estudio se plantea mostrar la importancia del desarrollo de una planeación estratégica en las organizaciones y la implementación de un adecuado plan de negocios que proporcione estabilidad y éxito en sus actividades disminuyendo el riesgo que generan los mercados actuales. Dichos mercados son cada vez más competitivos, exigentes y agresivos. Solo las compañías que mejor estructuradas estén podrán competir y crecer, solidificar sus bases y posicionarse en los mercados. El estudio es realizado bajo el método de investigación reflexiva. Este permite indagar información real del mercado proporcionando resultados detallados de la situación actual de las organizaciones, permitiendo generar nuevas directrices correctivas que permitan suministrar alternativas de solución a las organizaciones. Como resultado, se logró ampliar el panorama actual de las organizaciones y la problemática que enfrentan encuestando a tres, del sector de "Macrosnacks" que proporcionaron información fundamental para la generación de soluciones.

Palabras clave: competitividad, planeación estratégica, plan de negocios, sostenibilidad, diferenciación.

JEL: L1, L60,R58,R11, O31

\section{ABSTRACT}

Actually many small businesses are currently failing in the century 21 rst because of the lack of implementation of strategic planning and the lack of a business plan that allows them to chart the ways to achieve each of their goals and objectives. This situation is very worrying, because many companies cease their activities and end up going bankrupt damaging the economy and slowing down national development (century 21rst). It is for this reason that this research proposes to show the importance of the development of strategic planning in companies and the implementation of an adequate business plan that provides stability and success in their activities, reducing the risk generated by the current markets. These markets are becoming more competitive, demanding and aggressive. Only the companies that are better structured will be able to compete and grow, solidify their bases and position themselves in the markets. The research is done under the method of reflective research. This allows you to investigate real market information by providing detailed results of the current situation 
of companies, allowing the generation of new corrective guidelines that allow companies to provide alternative solutions. As a result, it was possible to expand the current vision of the companies and the problems they face by surveying three of the "Macrosnacks" sector that provided fundamental information for the generation of solutions.

Keywords: competitiveness, strategic planning, business plan, sustainability, differentiation.

JEL: L1, L60,R58,R11, O31

\section{RESUMO}

Atualmente, muitas organizações pequenas falham devido à falta de implementação do planejamento estratégico e à falta de um plano de negócios que lhes permita traçar as formas de alcançar cada um dos seus objetivos e metas. Esta situação é bastante preocupante porque, devido a isso, há muitas organizações que cessam suas atividades e acabam indo à bancarrota, prejudicando a economia e atrasando o desenvolvimento nacional. É por este motivo que este trabalho pretende mostrar a importância do desenvolvimento do planejamento estratégico nas organizações e a implementação de um plano de negócios adequado que proporcione estabilidade e sucesso em suas atividades, reduzindo o risco gerado pelos mercados atuais. Esses mercados são cada vez mais competitivos, exigentes e agressivos. Somente as empresas mais bem estruturadas poderão competir e crescer, solidificar suas bases e posicionar-se nos mercados. O trabalho é feito sob o método de investigação reflexiva. Isso permite que informações reais de mercado sejam investigadas, fornecendo resultados detalhados da situação atual das organizações, permitindo gerar novas diretrizes corretivas que permitem oferecer alternativas de solução para as organizações. Como resultado, foi possível ampliar o atual panorama das organizações e os problemas que elas enfrentam ao examinar três do setor "Macrosnacks", que forneceu informações fundamentais para a geração de soluções.

Palavras-Chave: competitividade, planejamento estratégico, plano de negócios, sustentabilidade, diferenciação.

JEL: L1, L60,R58, R11, O31 


\section{INTRODUCCIÓN}

Son muchas los empresarios que deciden iniciar una organización en el siglo XXI, pero pocos son los que logran sostenerlas con éxito en el tiempo y aumentar el valor de la misma, el siglo XXI es exigente. Esto se debe al desconocimiento de factores fundamentales que se deben tener en cuenta para garantizar el buen funcionamiento de la organización y su perdurabilidad. Es por ello que se plantea a continuación la importancia del diseño y la incorporación de un plan estratégico que evitará un panorama desfavorable de incertidumbre en los objetivos y metas. Según lo explica Torres (2006) la planificación hace referencia a trazar con anticipación las vías mediante las cuales la organización será encaminada para seguir de manera estratégica cada uno de los pasos que garantizará el crecimiento organizacional en el futuro. Es decir que la compañía depende de la planeación para reafirmarse y sostenerse en el futuro, así como un barco requiere de su timón para guiar su camino, de esta misma manera la organización requiere de la planeación y el control para ir por la vía correcta que garantice el éxito de la misma.

Los principales propósitos de la planeación estratégica radican principalmente en la elaboración de una guía que le permita trazar las vías mediante las cuales se alcanzarán cada uno de los propósitos de la organización en cada una de sus áreas permitiendo tener claros cada uno de sus objetivos y la manera como estos serán llevados a cabo. También permite tener un panorama claro del estado actual de la compañía y la toma de decisiones basadas en datos verídicos e información real. En la actualidad son muchas las organizaciones que subvaloran la importancia de tener planteadas unas estrategias de gestión que las direccionen por el buen porvenir. Por tal razón los directivos de las compañías deben constantemente retroalimentarse y actualizarse en nuevos métodos de gestión que vayan a la vanguardia de los nuevos requerimientos que el mercado exige; por otro lado, el cambio de mentalidad conservadora a una mentalidad más liberal y abierta al cambio, genera avances satisfactorios para las organizaciones. Es por esto, que según afirma Torres (2006) "la planeación estratégica es un proceso mental que realiza generalmente año a año el equipo directivo, con el objetivo de diseñar e implementar el plan estratégico que le permitirá enfrentar con éxito el cambio organizacional". 
En este sentido lo que se busca con el siguiente estudio es brindar ciertos puntos críticos en los cuales las organizaciones están fallando mayoritariamente a la hora de diseñar los planes estratégicos; y así empezar a generar una conciencia sobre la importancia de la correcta implementación de la planeación y como se ha convertido en la actualidad en un diferenciador de éxito organizacional.

\section{PLANTEAMIENTO DEL PROBLEMA}

Hoy en día la competitividad y la innovación constante son los diferenciadores entre las organizaciones exitosas y aquellas que no lo son.

Debido a los actuales cambios hoy en el siglo XXI están sufriendo los mercados a nivel mundial, que cada vez son más exigentes, se hace relevante la planeación estratégica como método de mitigación de riesgos de mercado y como estrategia de reingeniería constante y adaptable a las nuevas necesidades de los clientes. La planeación estratégica es un proceso que le permite a las organizaciones definir la misión, los objetivos principales, las estrategias sobre asignación de recursos, entre otros; con el fin de cumplir unas metas planteadas (visión) (Mejía Argueta, Agudelo, \& Soto Cardona, 2016). Es por estas razones, que se hace indispensable la estructuración de una gestión organizacional clara y concisa que les genere a las organizaciones a una mejor sinergia interna, convirtiendo una debilidad en una fortaleza, que le ayudará a afrontar el cambiante mundo de los negocios.

Acorde con Mejía Argueta et al.

Las compañías deben abandonar la idea de que la efectividad operacional es suficiente para sobresalir en el mercado y en lugar de ello enfocar esfuerzos en realizar procesos de planeación estratégica que les permita definir coherentemente las acciones y tácticas (2016).

Aquella organización que actualmente no esté pensando en mejorar, innovar, actualizar los modelos de organizaciones antiguas y aún más importante, plantear estrategias que la lleven a ser una compañía preparada y exitosa; sufrirá las consecuencias de la globalización. 
Actualmente la planeación estratégica se ha convertido en un componente necesario de la gestión organizacional; es por esto que Mintzberg (1994) estipula cuatro componentes que describen la importancia de la planeación:

1. Las organizaciones deben planear para coordinar sus actividades.

2. Las organizaciones deben planear para asegurarse que se tiene en cuenta el futuro.

3. Las organizaciones deben planear para ser racionales.

4. Las organizaciones deben planear para controlar.

A medida que las organizaciones no acepten que los mercados ya no son los mismos de hace diez años y no creen estrategias y métodos de gestión cada vez más inteligentes y estructurados, irán quedando en el pasado y perdiendo poco a poco mercados; y en la situación más extrema el mercado las sacará totalmente llevándolos a la ruina y liquidación de la organización.

\section{JUSTIFICACIÓN}

Cuando una organización busca diferenciarse de los demás, necesita hacer cosas nuevas. Ya sea que la organización se vaya a fundar o busque adaptarse, consolidarse o desarrollarse en un mercado (Hernández y Rodriguez, 2011), tendrá que evaluar a la competencia y buscar la manera de distinguirse de esta. Tendrá que pensar en las estrategias que va a usar en el futuro con mayor rapidez que lo hace la competencia toda vez que entienda que el mercado cambia constantemente junto con las necesidades de los clientes (Porter, 1990). Si una organización decide prescindir de una planeación estratégica, estará yendo ciega por el camino. Gran parte del éxito de una compañía es definir unas estrategias claras que conlleven a conseguir los objetivos.

Es importante hacer ver que la planeación estratégica en el siglo XXI es uno de los factores fundamentales a la hora de justificar decisiones a largo plazo (Hernández y Rodriguez, 2011), por tal razón se hace plenamente necesaria para todas las organizaciones que desean incursionar en otros mercados y mucho más indispensable en los mercados internacionales. La planeación estratégica guía y 
pone un horizonte a las compañías. Una misión bien hecha amplía la visión (Ver figura 1), es decir no la limita y esta a su vez es la encargada de mantener unido a un equipo de trabajo.

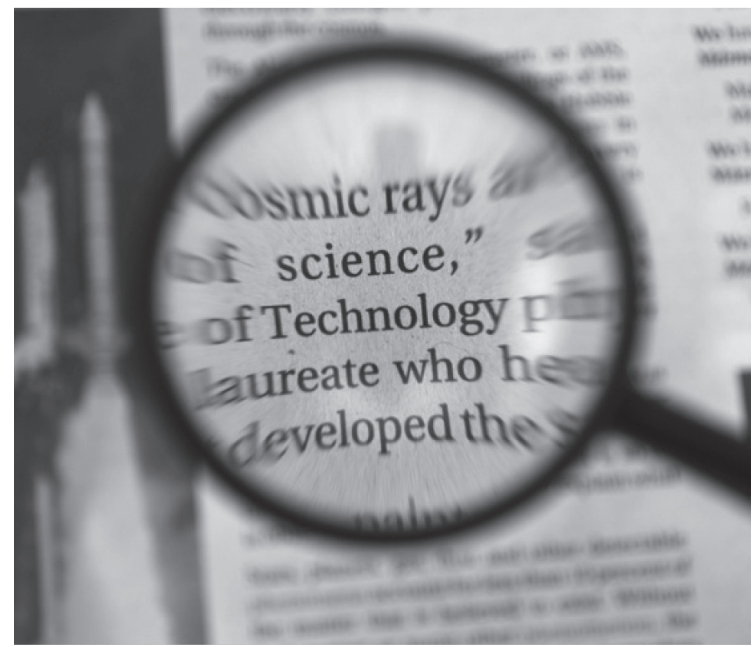

\section{Figura 1}

Ampliación de una perspectiva.

Fuente: Imagen de Public Domain Pictures en Pixabay

La visión es el proceso mental encargado de motivar a las personas, de hacerlos sentir que trabajan por una sola causa por lo que, si se quiere trabajar como equipo de alto rendimiento, la organización deberá tener en mente estas ideas para poder dirigirla exitosamente. Es decir, el éxito de una compañía radica en su nivel de competitividad o en el nivel de efectividad con que la organización satisface a sus clientes. La competitividad necesita un marco de referencia, este es dado por la misión y la visión de la entidad, si la organización no cuenta con estas, estará por debajo de la competencia.

Una organización que entre sus ideas rectoras quiera incursionar y posicionarse en los mercados internacionales deberá estar guiada por un plan de acción. El plan de acción deberá ir enfocado en mantener o aumentar las ventajas competitivas de la organización, por este motivo no se puede elaborar un plan de acción sin haber encontrado las ventajas internas de la organización que a su vez se logran gracias a la existencia de una planeación estratégica. 
Por último, existen organizaciones que ya poseen estrategias para su direccionamiento; sin embargo, llevarlas a cabo tiende a ser confuso y complicado. Estas organizaciones, bajo su opinión no necesitan de una planeación estratégica. Incluso argumentan que esta les produce salidas excesivas de dinero. Con mayor razón es cuando deben considerarla porque muchas veces lo único que necesitan es mayor claridad a sus estratégicas, mejorar sus tácticas y la forma como hacer que funcionen. Entonces podrán apoyarse en la planeación estratégica.

\section{MARCO TEÓRICO}

\section{Desorden interno por desconocimiento de las metas y objetivos.}

Las organizaciones que pretenden incursionar en el mercado internacional, deben buscar una coordinación en sus actividades (Porter, 1990). Pero además como dicen los autores Armario, Rastrollo, \& González Robles (2009) debe existir una gestión del conocimiento ya que de acuerdo con González-Campo \& Hurtado Ayala (2014) esto facilita una mayor actividad innovadora y en consecuencia una mayor cohesión del equipo en sus labores. La organización no se maneja únicamente por la suma de unos procesos. Sin la coordinación de los mismos, la cadena de valor se descompone y puede provocar el incumplimiento de metas (Ochoa Díaz \& Ríos Millán, 2011). La compañía deberá identificar y comunicarle a todo su equipo cuál es su punto fuerte y mantenerlo en el tiempo. Tanto si una organización busca destacarse por sus bajos costes o por un producto diferenciado deberá estar en sincronía con todas las personas que laboran allí. Sus colaboradores al interior de la organización deben saber que, si la estrategia es de bajos costes, la eficiencia en sus tareas es primordial. Y si se trata de diferenciación es indispensable el cuidado, la precisión y el control para un producto de máxima calidad.

El desconocimiento del objetivo principal en una organización, hace que la misma pierda el rumbo. Esto debido a que los objetivos juegan un papel fundamental en la dirección de la organización. Estos revelan la posición de la organización frente a su sector, es decir la importancia de una compañía dentro de un sector depende de la magnitud de los mismos (Porter, 1990). Si la compañía al contrario no sabe cuál es la dimensión de lo que quiere alcanzar o por el contrario lo quiere alcanzar todo, al final no va a obtener mucho como organización mundial. 
Tampoco conseguirá poner un orden al interior en sus diferentes departamentos. Las organizaciones deben determinar claramente en la planeación estrategia su punto de partida y en la medida de la tecnología que poseen, el recurso humano y nuevas ideas del horizonte realista de la organización. El entorno empresarial es muy competido debido a que cuenta con tecnología de punta y procesos únicos, esto hace que las empresas realicen reingeniería en los procesos, probablemente ponerse metas muy altas le sea contraproducente y frustrante.

El orden en una organización tiene una importancia en sus actividades dado que radica en los objetivos que se hayan planteado (Porter, 1990). Por ejemplo, una compañía que se dedique a vender insumos para la industria y que el elemento final de su canal de distribución sea un mayorista no necesita hacer marketing en televisión y en radiodifusión. Esto es relevante en aquellas que venden al consumidor final y no en organizaciones que proporcionan insumos. Hacer esto solo provocaría una salida innecesaria de dinero. La difusión por estos medios solo impactaría al consumidor final; esto no quiere decir que las organizaciones se limiten a lo dicho. Si realmente se desea incursionar en el cliente final se debería abrir la posibilidad de vender productos finales para poder aplicar estrategias de mercadeo y fidelización de clientes (Ochoa Díaz \& Ríos Millán, 2011). La planeación en la organización le permitirá decidir el orden interno de la misma. Por lo tanto, la que desconozca su rumbo, hará esfuerzos en sentidos contradictorios. Eso puede llamarse desorden interno. Cada departamento debe estar organizado en razón de ser de lo que la compañía quiere conseguir y de esta forma elaborar los presupuestos. Por lo tanto, la estructura organizativa, es un reflejo de los objetivos de la misma.

La coordinación del equipo de trabajo, conduce hacia el orden interno (Ver figura 2). La gestión de un buen líder le permite a la organización altos desempeños. El gerente debe tener la capacidad de controlar y permitir la participación de todo el equipo en el desarrollo de nuevas ideas para construir ventaja competitiva (Escandon-Barbosa \& Hurtado-Ayala, 2016). 


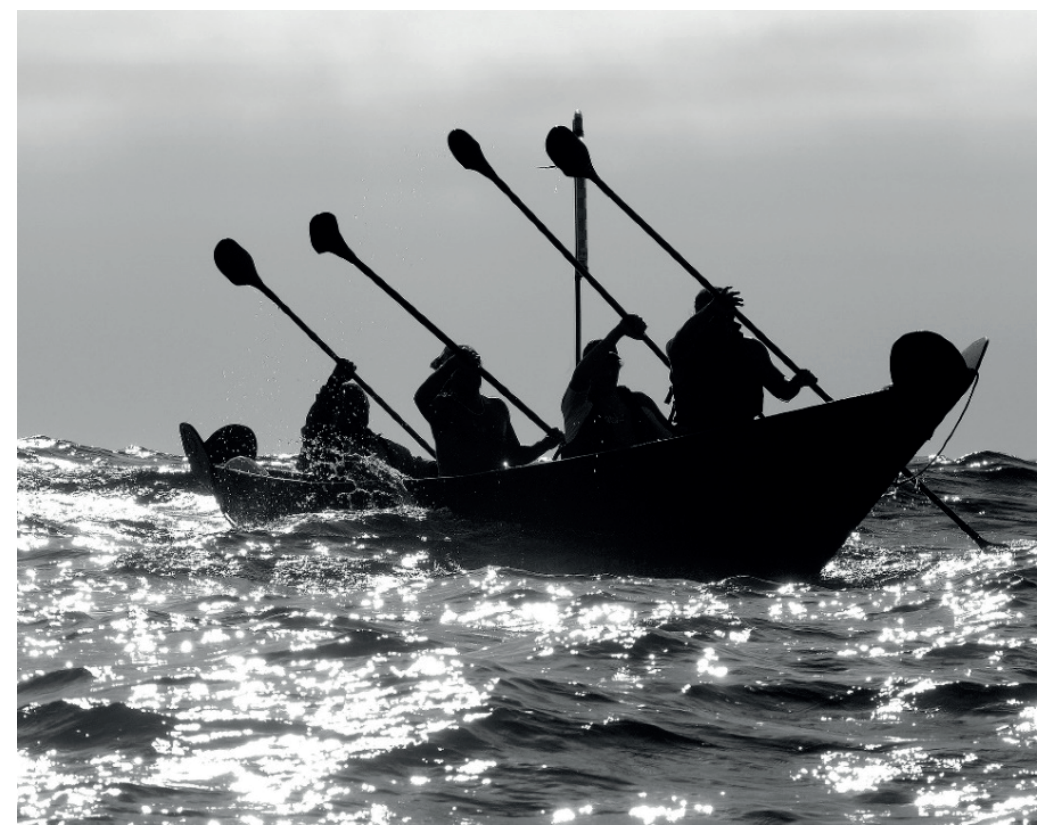

Figura 2

Trabajo en Equipo

Fuente: Imagen de skeeze en Pixabay

Incertidumbre en el futuro de las organizaciones por desorientación en la visión.

La visión en una organización debe estar escrita y bien definida. Si esto no se hace así, puede disminuir la productividad de la misma y aumentar el riesgo de estabilidad en su sector. El riesgo es evidente ya que cada paso que de la organización lo hará sin timón. La visión es el punto de partida para crear los objetivos. Una compañía debe tener claro su horizonte, y el nivel que quiere alcanzar. Por ejemplo, si se quiere ser una organización representativa en su sector (una visión más clara) debe revisar la calidad de sus productos (Botello peñaloza, 2016) y la de su competencia y buscar sobresalir en lo que las demás de su sector no ofrecen como valor agregado en sus productos.

El lugar que la organización quiere adjudicarse en un sector depende de una visión clara y objetiva. Si se quiere competir a nivel internacional debe tener claro el mercado que va a atacar y hacer una buena gestión de riesgo. La organización no 
se puede expandir y dejar mercados insatisfechos. No puede hacer exportación sin haber definido el canal de distribución, el segmento de mercado, sectores afines donde también competirá y zonas geográficas que atenderá. La compañía debe atacar en la medida de sus posibilidades y no pretender competir con precios bajos cuando existen gigantes en su sector con productos similares que podrían sacarla del mercado rápidamente (Porter, 1990). Como ya se dijo anteriormente, si la organización que desea abrirse paso en el mercado internacional no encuentra su verdadera ventaja competitiva y cree ser buena en todo va a terminar fracasando debido a las contradicciones que cada estrategia posee.

Alcanzar la visión o el éxito organizacional debe ser la definición misma de esta. Es decir, reducir el riesgo de incertidumbre en el futuro de una organización solo podrá ser si basa sus estrategias en la mejora constante de sus procesos de innovación. Solo si la organización comprende su entorno de manera objetiva y descubre la manera de como aportar para que cada día sea mejor, estará visualizando el futuro acertadamente y proporcionando más fuertes bases para trascender en el tiempo. Es importante que los objetivos en pro de alcanzar la visión de una organización hayan sido diseñados con el fin de solucionar problemas globales. Si la organización solo soluciona problemas internos y no problemas en el extranjero verá reflejado este error en las ventas del producto en el exterior y en consecuencia poniendo en riesgo su estabilidad.

\section{Perdida de dinero en las organizaciones por una inadecuada gestión financiera.}

La gestión financiera es una de las áreas más importantes para el funcionamiento de cualquier entidad, ya que se encarga de la administración de los recursos económicos que tienen las organizaciones para asegurar la cobertura de los gastos; de esta manera se pueda llevar un control adecuado y ordenado de los ingresos y salidas de dinero. Es por esta razón que el grado de sensibilidad al error en esta área es muy alto y la inadecuada planeación financiera se podría traducir en grandes pérdidas monetarias.

Según Calvo Vérgez (2011) la planeación financiera cuantifica los recursos que serán utilizados en la realización de proyectos, es por esta razón que la organización debe proyectar financieramente cada recurso a utilizar en el 
presente pero también en el futuro. De esta manera, en adelante garantizará la viabilidad del proyecto cuando se incursione en el mercado y contará con los recursos financieros suficientes para la realización del mismo cumpliendo con los objetivos propuestos de manera efectiva, eficaz y satisfactoria.

En la construcción de un panorama estable que proporcione condiciones óptimas para la generación de un adecuado ambiente de gestión financiera se deben tener en cuenta factores importantes como: Una buena cultura financiera que optimice el uso de los recursos financieros, un constante aprendizaje y actualización de aprendizaje que permita adaptarse a los cambios y un eficaz manejo de la información que permita tener actualizada cada una de las áreas para la toma de decisiones (González Ramírez, 2001).

Particularmente el último elemento mencionado, es el componente central del sistema de información financiera, porque comprende y aporta componentes que permiten una óptima planeación, control, medición y seguimiento del impacto que tienen las decisiones tomadas por la gerencia referentes al correcto funcionamiento de la organización.

Es importante resaltar que cada mejora, por pequeña que sea, puede fortalecer significativamente el desempeño general, y la inadecuada gestión de la misma se podría traducir en pérdidas significativas. Por tal motivo actualmente los gerentes financieros deben incrementar sus habilidades para desarrollar procesos de transacciones y actividades de control, como la solución de problemas, la administración del riesgo, el análisis de procesos de negocios, los conocimientos técnicos, la orientación al cliente, la formación tecnológica, la construcción de relaciones y equipos, habilidades de comunicación, la administración del cambio, el conocimiento estratégico, liderazgo y negociación (Meza Orozco, 2010; Martinez Martinez \& Fernandez Hurtado, 2018)

\section{Importancia de un plan de negocios para la determinación de objetivos y metas de las organizaciones.}

Un plan de negocios es una herramienta que permite a las organizaciones trazar una ruta por medio de la cual alcanzarán cada uno de sus objetivos y metas propuestas, esta herramienta permitirá abordar cada uno de los obstáculos 
esperados y no esperados que se podrán presentar en el futuro. Además, es una herramienta de gran utilidad que le permite a una organización determinar qué tan competitiva puede ser en el mercado.

El plan de negocios es una herramienta que permite reunir en un solo documento toda la información de una organización, brindando un panorama de la viabilidad del negocio durante su preparación, además de que permite evaluar, implementar lineamientos, buscar alternativas y proponer planes de acción que ayudaran a la puesta en marcha el negocio (Alvarez Torres, 2006).

La información cuantitativa debe estar sustentada en propuestas estratégicas, comerciales, económicas, financieras, de operaciones y de recursos humanos. Los objetivos que justifican la elaboración de un plan de negocios difieren según el momento de la vida de la organización y el tipo de negocio que vaya a planificarse.

En el plan de negocios se deben establecer cada una de las metas de corto mediano y largo plazo que espera alcanzar la organización estas metas deben ser trazadas con objetivos concretos y específicos que permitan a cada uno de los involucrados en las mismas desarrollarlos de tal manera que haya una articulación entre los diferentes departamentos involucrados. Debemos tener en cuenta que los objetivos son situaciones o estados que una organización espera alcanzar en un determinado laso de tiempo y con los recursos que cuenta en su momento o con los recursos que esta espera obtener.

Determinar objetivos es de gran importancia ya que estos definen el éxito de los mismos además trazan el curso y los lineamientos de cómo serán alcanzados y motivan a las personas que integran la organización (Fernandez, 2014). Estos objetivos permiten que las actividades y esfuerzos grupales sean enfocados en un mismo propósito y dirección optimizando los recursos utilizados y disminuyendo el tiempo utilizado para alcanzar los objetivos propuestos.

Un plan de negocios es una herramienta de la planeación estratégica que le permite a la compañía determinar el futuro de la misma es decir planear el futuro sin esperar a saber que le depara el destino a su organización. Algunos de los beneficios que proporciona el elaborar un plan de negocios bien estructurado son: 
1. Focalizar las mejores oportunidades de negocio de la organización: al tener un buen plan de negocios bien estructurado y definido podemos obtener información precisa acerca de las capacidades con las que cuenta la organización de este modo podemos saber con precisión cual es el alcance de la misma y determinar en qué negocios podemos incursionar.

2. Crear una base firme que permite establecer y trazar todas las actividades de la organización: el plan de negocios crea una base de datos precisa y organizada de todas las actividades que en la compañía se realizan de este modo es más versátil establecer futuras actividades que la organización realizara.

3. Manejo más eficiente de los recursos: el plan de negocios suministra un mayor orden en cuanto a los recursos que posee la compañía además de información detallada de cada uno de ellos esto proporciona un manejo más eficiente y productivo de los mismos.

4. Crea claridad sobre los obstáculos que se podrían presentar: el plan de negocios brinda información que permite esclarecer cada una de las barreras a las que se podrían enfrentar los objetivos y metas de la organización permitiendo elaborar planes de contingencia para sobrepasar dichos obstáculos de manera satisfactoria y sin contratiempos.

5. Determina que áreas de la organización se encuentran afectadas y requieren de un mayor control: el plan de negocios incluye un diagnostico general de toda la compañía que permite identificar las falencias que se presentan en cada área y efectuar su correspondiente corrección.

\section{Perdida de mercados potenciales por limitaciones en la expansión.}

En la actualidad muchas organizaciones se comportan de manera conservadora y por desconocimiento se privan de explorar nuevos mercados y expandir sus alcances. Esta situación puede llevar a estas organizaciones a disminuir drásticamente sus ventas hasta ser cerradas a causa de no incursionar en nuevos mercados y ser parte de la creciente globalización que experimenta el mundo. 
Es por esta razón que las organizaciones deben percibir la globalización como una realidad creciente y latente a la cual deben integrarse y ser parte de esta, cada día es más común y se debe tener en cuenta para alcanzar el éxito organizacional. La globalización y la exploración de nuevos mercados son dos factores de suma importancia los cuales deben ser integrados en las metas principales de una compañía estas metas deben trazarse (Hurtado, 2013), pero antes hay que preparar a la organización para enfrentarse a estos retos por medio de un planeación estratégica bien estructurada y definida que le permita contar con las competencias necesarias para ofrecer un producto que cuente con los márgenes de calidad requerida por los nuevos mercados.

Algunas de las razones y beneficios que brindan la apertura de nuevos mercados son:

Mantener un crecimiento sustentable del negocio: La exploración de nuevos mercados se encarga de expandir los clientes y por ende incrementar la demanda y ventas de la organización esto representa ingresos adicionales para la misma.

Mantener una ventaja competitiva: la expansión a nuevos mercados incentiva a la organización para mejorar la calidad del producto que ofrece es por esta razón que las organizaciones deben alcanzar estándares de calidad óptimos y brindar a su producto un valor agregado que le permita competir con los diferentes actores del mercado esto conlleva a que la organización se exija a sí misma en cuanto a calidad y mejoras en sus productos haciéndolas altamente competitivas.

Superar épocas de crisis: cuando una compañía distribuye su participación en variados escenarios o mercados se cubre ante el riesgo de no tener todas sus ventas dirigidas hacia un solo mercado así cuando el comportamiento de un mercado no es muy favorable los otros mercados se comportan de manera contraria contrarrestando su efecto negativo y disminuyendo el riesgo de pérdidas.

Cuando una organización toma la iniciativa de incursionar en nuevos mercados internacionales debe enfrentarse tanto a barreras internas como externas dichas barreras deben ser mitigadas mediante un adecuado estudio de mercado que permita la elaboración de estrategias de marketing que faciliten dicho proceso y que permitan sobrepasar cada una de las barreras tanto internas como externas a las cuales la organización se verá enfrentada (Martínez Carazo, 2010). 
Las barreras internas hacen referencia principalmente a carencias de recursos dentro de la organización tales como: recursos humanos con capacitación suficiente, calidad del producto que brinde un mayor valor agregado, innovación tanto en el producto como su empaque, recursos financieros suficientes que den vía libre al proyecto, información entre otras. Debido a la falta de estos factores la estrategia de marketing se ve afectada generando inconvenientes en el proceso de internacionalización de la compañía (Prieto Herrera, 2009).

En cuanto a las barreras externas; estas se ubican en el entorno nacional e internacional y hacen referencia a: canales de distribución, competencia, impuestos, demanda de consumidores entre otras. Estas puede afectar de manera directa el plan de internacionalización de la organización y son barreras las cuales no puede controlar la compañía pero si puede preverlas y estar preparada para mitigarlas mediante planes de contingencia que permitan la continuación de cada uno de los proyectos planteados (Rozas Gutiérrez et al., 2013).

\section{METODOLOGÍA}

El modelo de estudio que se implementará es reflexivo y correlacional teniendo en cuenta que este tipo de investigación se dedica a la obtención de procesos de organización en un sector dado (Sánchez, 2012) y su objetivo es recoger información para revelar nuevas señales por medio de la indagación (Lerma González, 2009) de acuerdo a unas variables de medición determinadas (Ver tabla 1). Que podrán brindar al lector un panorama que recree la importancia de la planeación estratégica y mostrar un diagnostico general de cómo está la compañía en cuanto a sus objetivos y metas para que puedan ser efectuadas sus respectivas correcciones.

La población a la cual estuvo orientada esta investigación es hacia el entorno empresarial, que tienen bajo indicador de eficiencia en las relaciones interpersonales y que urgen de una herramienta que genere cambios positivos tanto en la organización como en todos sus colaboradores.

Se aplicaron 135 encuestas en donde se aplicó el método estadístico para determinar la muestra de población finita (ver la siguiente formula), en donde se obtuvo una muestra de 112 de acuerdo al nivel de confianza $(Z)$ del $96 \%$ y con un margen de error (e) del $4 \%$. 


$$
\frac{N^{*}\left(\mathrm{a}_{c} * 0,5\right)^{2}}{1+\left(e^{2} *(N-1)\right.}=
$$

$\alpha=$ valor del nivel de confianza (varianza)

$\mathrm{e}=$ Margen de error

$\mathrm{N}=$ Tamaño de población

De acuerdo a la ejecución de los instrumentos de recolección se tabularon las encuestas mediante la elaboración de bases de datos y tablas de frecuencia, utilizando la herramienta Excel y STATGRAPHICS; para la explicación de la información se realizó enfocada en precisar las diferentes variables que inciden dentro del clima organizacional y en la eficacia de las mismas aplicadas a las empresas.

Este modelo busca explicar la importancia de la implementación de un plan estratégico a nivel interno de la organización, pero considera también que la compañía por tener conexión con el ambiente externo, este último es importante para la planeación de estrategias (Mendoza, 2012). El modelo también muestra los beneficios que se podrían obtener si se elabora una correcta planeación disminuyendo los riesgos innecesarios y aumentando el grado de éxito en el alcance de los objetivos de la organización.

\section{ANÁLISIS DEL ESTUDIO}

A continuación, se comparan las opiniones acerca de la importancia de la planeación estratégica para algunas organizaciones del sector de Macrosnacks. Estas encuestas fueron realizadas con preguntas cerradas a manera de reflexión, con lo cual se sigue la metodología del presente estudio. 


\section{Tabla 1}

Correlación y desviación estándar de variables.

\begin{tabular}{|c|c|c|c|c|c|c|c|}
\hline Variables & $\begin{array}{c}\text { Mean- } \\
\text { Promedio }\end{array}$ & $\begin{array}{l}\text { Desviación } \\
\text { estándar }\end{array}$ & 1 & 2 & 3 & 4 & 5 \\
\hline $\begin{array}{l}\text { Adecuada } \\
\text { infraestructura } \\
\text { laboral }\end{array}$ & 0,72 & 0,45 & 1 & & & & \\
\hline $\begin{array}{l}\text { Estrategias } \\
\text { organizacionales }\end{array}$ & 0,79 & 0,41 & 0,17 & 1 & & & \\
\hline $\begin{array}{l}\text { Desempeño } \\
\text { organizacional }\end{array}$ & 0,82 & 0,38 & 0,12 & $0,08 * * *$ & 1 & & \\
\hline $\begin{array}{l}\text { Eficiencia } \\
\text { laboral }\end{array}$ & 0,84 & 0,37 & 0,22 & $-0,09^{*}$ & 0,01 & 1 & \\
\hline $\begin{array}{l}\text { Ideas } \\
\text { innovadoras }\end{array}$ & 0,83 & 0,38 & 0,19 & 0,03 & $0,07 * *$ & $0,07 * *$ & 1 \\
\hline
\end{tabular}

Fuente: Elaboración propia (2018)

Cuando se habla de internacionalización de las organizaciones, la planeación estratégica toma gran relevancia ya que se vuelve un elemento diferenciador y de éxito organizacional (Fernandez, 2013; Fernandez Hurtado \& Martinez Martinez, 2017). Al preguntar sobre este rubro, sobresale la gran importancia que los empresarios le dan a este punto, llegando a la conclusión de que es muy importante realizar la planeación estratégica para no cometer errores ni perder dinero a la hora de exportar un producto; funciona muy bien para expandir la organización. En otra de las respuestas dadas por una de las compañías se deduce que permite de manera ordenada lograr identificar los puntos fuertes de la organización y le permite posicionarse en su sector.

Observando la tabla de variables con sus respectivos resultados y cruces, muestra que la variable con mayor correlación es la variable de estrategias organizacionales con respecto a la variable de desempeño organizacional en donde muestra un rango del 0.08 de correlación entre sí con un mean- promedio de 0,82 y con una desviación estándar del 0,38 dado a que este nivel se nos aproxima 
significativamente a +1 es bastante bueno y muestra que las dos están ligadas a generar eficiencia laboral empleando ideas que se encaminen hacia la innovación y mejora constante, así como también obtener un plan de calidad en el cual se pueda medir periódicamente la evolución que se tiene cuando se implementa alguna estrategia con el fin de superar falencias que se hayan detectado y con las cuales las organizaciones mejoren y presenten un mejor desempeño en el sector en el cual está desarrollando su función comercial o industrial, iniciando a partir de una óptima y adecuada infraestructura, contando con los equipos necesarios, en condiciones aptas para el desarrollo de lo anteriormente mencionado, ya que de esta manera afecta directamente la motivación del personal y es muy importante empezar por un ambiente de higiene y pulcritud para llevar a cabo las actividades laborales que va de la mano con la implementación de un sistema de seguridad y salud en el trabajo que modera y regula todos los hechos y acciones que ocurren dentro de este sistema.

La siguiente variable a destacar es la variable de desempeño organizacional con la variable de ideas innovadoras con rango de 0,07 lo cual indica un nivel normal de correlación con un mean-promedio de 0,83 y una desviación estándar de 0,38 el rango de correlación de estas variables se aproxima a +1 por ende son de gran importancia para las organizaciones dado a que conjuntamente conllevan a la organización a tener un desempeño óptimo en el posicionamiento de sus habilidades corporativas para que de esta manera se cumplan con las estrategias organizacionales con una alta eficiencia laboral implementando prácticas y estrategias que estén a la vanguardia con respecto a la competitividad del mercado, esto conlleva a tener un plan a ejecutar con un único fin que es lograr cumplir las metas que se proponen y que de esta manera lleven a la empresa a la cúspide de su desempeño organizacional logrando posicionarse en el top con respecto a su competencia. Las empresas hoy en día deben estar en constante cambio positivo, absorbiendo toda la información, herramientas y estrategias que se implementan al día a día, siendo un objetivo que se mide a diario cuando se lanza un nuevo producto o servicio que pueda optimizar procesos y generar cambios para bien de la empresa mejorando la eficacia que se ve reflejada en la consecución del logro en cada factor diferenciador que diferencia a una empresa.

Siguiendo el análisis la variable de eficiencia laboral con la variable de ideas innovadoras obtienen un rango de 0,07 el cual indica un nivel normal de 
correlación con un mean- promedio de 0,83 y una desviación estándar de 0,38 cuyo rango de correlación de estas dos variables se aproxima a +1 son relevantes debido a que se complementan de una manera efectiva en cuanto al crecimiento y posicionamiento de la organización, para que de esta manera genere un nivel alto de eficacia laboral por las cuales las grandes empresas obtienen altos márgenes de rentabilidad en el momento en que se va a llevar a cabo un contrato nuevo, los indicadores de eficacia son de suma importancia para tomar la decisión de tomar los servicios o no de dicha empresa ya que muestra de una manera directa que tanto alcance se tiene en cuanto al cumplimiento y trazabilidad de los puntos o factores que inciden en dicha eficacia. Esto va de la mano con el cumplimiento de las estrategias implementadas por la organización y por ende genera un clima organizacional bastante óptimo en todos sus departamentos en operación cuando se establece una meta y esta se cumple con la colaboración de todo un equipo de trabajo que se motiva por alcanzar sus proposiciones de eficiencia empleando métodos de innovación y que a su vez este genere un impacto positivo para la empresa.

En la siguiente variable ponderada, la correlación entre eficiencia laboral y estrategias organizacionales presenta un rango de $-0,09$ con el cual se observa un nivel bajo de correlación con un mean-promedio de 0,84 y una desviación estándar de 0,37. De acuerdo al rango presentado por estas dos variables se determina que las variables presentan complejidad en el desempeño individual de cada colaborador frente a las estrategias formuladas y desarrolladas por las organizaciones. Esto Lleva a que el rendimiento en conjunto de toda la estructura organizacional dependa de la excelente formulación de estrategias por parte de la junta directiva o gerente general y logren aplicarlas a todo el esquema organizacional, pero que además de implementarlas se logre tener una comunicación de manera asertiva que se vea reflejado en el ambiente laboral cuando se establezca un plan de trabajo, así cada persona se verá más involucrado y tenga más sentido de pertenencia a nivel organizacional. La correlación siendo negativa es inquietante ya que es un problema al cual se le puede dar una solución de manera eficaz, al aplicar métodos comunicativos y de liderazgo para que las relaciones laborales afecten positivamente los indicadores de eficiencia y desempeño en cada una de sus áreas, lo que hace referencia a un constante evaluador de desempeño y acompañamiento para medir el alcance que se tiene por parte de cada individuo y por el cual depende la empresa de su labor. 
Por otro lado, cabe resaltar que si bien es importante que las empresas inviertan en el desarrollo de sus empleados, puesto que esto aumenta su habilidad y eficiencia en la realización de tareas y toma de decisiones, y se obtiene un talento humano con más habilidades y capacidades que garantice la satisfacción del cliente final. Las Organizaciones las cuales salvaguarden el impacto de los factores internos y externos a sus colaboradores, lograran obtener un buen desempeño de su grupo de trabajo de tal manera lograran una alta eficiencia en su desempeño y toma de decisiones referente a la posición, en que cada colaborador desempeñe dentro de la organización. Por ende la organización lograra un buen posicionamiento referente a las demás organizaciones las cuales carecen de herramientas para ejercer dichos manejos.

El manejo de las relaciones interpersonales en el ámbito interno conlleva a las organizaciones a formular y aplicar factores de manejo y convivencia referentes a un buen ambiente laboral donde se resalta: tener medios de comunicación muy bien acoplados entre sus departamentos. Mientras que en el ámbito externo la organización podrá formular una serie de incentivos los cuales mantengan a su personal motivado y de tal manera generar en ellos un alto sentido de pertenencia.

Por otro lado, la inexistencia de un plan estratégico podría perturbar la sostenibilidad de la organización en el tiempo, debido a la incertidumbre que generaría en los objetivos y metas de la compañía.

En cuanto a los problemas existentes sobre gestión organizacional, son muchas las dificultades que se vienen a la cabeza; pero se evidenció entre los entrevistados $\mathrm{y}$ entrevistadores una congruencia sobre los principales problemas por los cuales las organizaciones fallan, las cuales son: desorden interno, desorientación en la visión, inadecuada gestión financiera, inexistencia de un plan de negocios, limitaciones en la expansión de mercados. Siendo la falta de un plan de negocios uno de los puntos donde mayores falencias hay.

El plan de negocios es una herramienta que sirve para estructurar, evaluar y alinear una idea de negocio. Permite analizar y valorar la viabilidad inicial de una idea, además de servir como "carta de presentación" para la búsqueda de financiamiento, de nuevos socios e inversionistas (Ávila Cifuentes, Félix Antonio, \& Andrés Yesid, 2008). Es por ello que se convierte en el punto de 
partida para un buen planteamiento estratégico, ya que brinda la situación actual de la organización y a que se puede enfrentar teniendo en cuenta las ventajas competitivas y fortalezas con las que se cuentan.

Partiendo de la anterior premisa se evidencia que uno de los entrevistados no contaba con un plan de negocios, situación que dificulta su posible éxito organizacional, además de que genera una apariencia y sentimiento de informalidad ante los ojos del mundo organizacional. Actualmente esta situación en países como Colombia aún se ve en gran cantidad, mayoritariamente en las Mipymes por su poco conocimiento sobre el tema.

A continuación, se muestra una gráfica que muestra los niveles de inversión de organizaciones de Cali y Yumbo sean formales e informales entre los diferentes factores diferenciadores de las compañías.

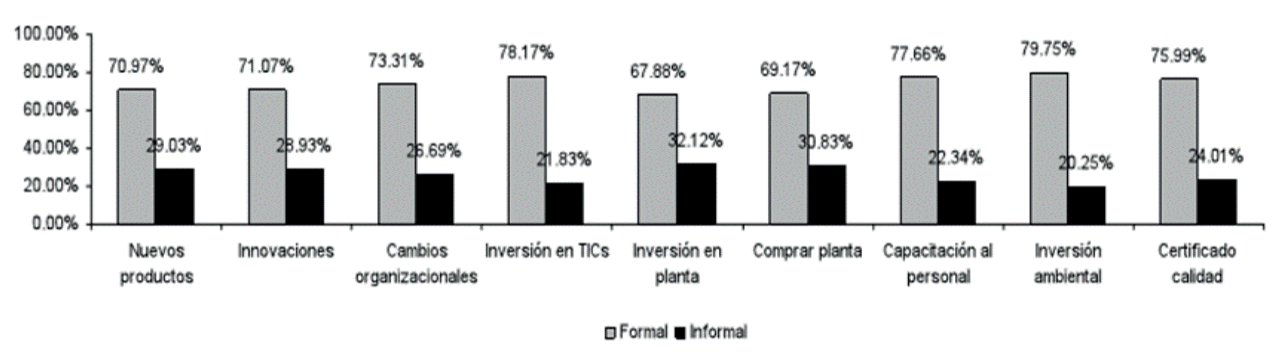

\section{Figura 3}

Distribución de innovaciones e inversión por tipo de organización Fuente: Censo de Cali y Yumbo 2015

Como se puede observar en el anterior grafico se evidencia que la inversión en las diferentes áreas claves de las organizaciones es mayor en aquellas que son formales, debido a que cuentan con una mayor organización interna y con un conocimiento de sus fortalezas y debilidades, información que un plan de negocios brinda y que un plan de acción puede solucionar (Cárdenas \& Rozo, 2009).

Según Blanco Gracía \& Segovia Vargas (2014) la planeación financiera es otro de los factores de gran importancia de la planeación estratégica ya que este garantiza el alcance y culminación de cada uno de los proyectos que sean trazados 
por la organización midiendo y pronosticando los recursos que serán utilizados para que las metas y objetivos sean llevados a cabo. Este factor determinara además de la cantidad de recursos que utilizara la compañía también el modo como serán obtenidos. Al cuestionar a los empresarios sobre este factor algunos manifestaron que dicha planeación era llevada a cabo, pero a corto plazo lo que generaba incertidumbre en la manera cómo iban a ser culminados los proyectos futuros de la compañía esto se debía a la falta de una inadecuada implementación de la planeación estratégica en sus organizaciones.

Otros de los factores evaluados y de gran importancia hoy en día en el mundo organizacional son el conocimiento de las ventajas competitivas con las que cuenta las organizaciones y las oportunidades que brinda el mercado. Al cuestionar a los empresarios sobre el conocimiento de las ventajas competitivas con las que cuentan cada una de sus organizaciones respondieron de manera similar afirmando que definitivamente cada una de sus compañías cuenta con un producto de alta calidad, diseño de empaque llamativo que le permite mayor durabilidad al producto. Al ser un bien de consumo se convierte en un producto de alta rotación lo que genera una producción continua y de fácil venta al público. La organización cuenta además con una página en dos idiomas, por medio de la cual se realizan ventas online.

De acuerdo a las entrevistas hechas, las organizaciones reconocen la importancia de una planeación estratégica. Pero además también reconocen que no le dedican el tiempo que deberían. Los empresarios coinciden con Porter (1990), cuando habla de que la planeación estratégica le permite ubicarse en el sector. Sin embargo, no toca el tema trascendental que es mantenerse en el tiempo.

\section{CONCLUSIONES}

Es fundamental en una organización la realización e implementación de un plan estratégico sin importar el tamaño o la razón social de la misma, ya que una adecuada implementación de un plan estratégico puede determinar el éxito o fracaso de la compañía. Así como un barco no puede navegar a la deriva o sin un propósito, una organización no debe existir sin un rumbo claro y conciso, debe tener bien estructurados sus objetivos y metas y la manera como alcanzarlos; es de suma importancia que la organización conozca su posición actual y la posición en la cual quiere estar en el futuro. 
Antes de incursionar en actividades de exportación la organización debería evaluar si es necesario hacer una reinvensión o una reingenieria de su compañía. Dificil es cuando el orden ya esta montado y se han invertido muchos recursos. Sin embargo existen formas de generar mayor valor agregado tales como: diseño de nuevos empaques que funcionen para varios mercados, mayor coordinación de procesos; en resumen mayor inversión en I+D (Porter, 1990) que podrían convertirse en ventajas competitivas que desembocarían en indices de rentabilidad mayor y de sostenibilidad de la organización. Si la organización sabe buscar, encontrará por medio de la $\mathrm{I}+\mathrm{D}$, información acerca de las necesidades que los clientes poseen y nuevas técnicas de comercialización. Con el tiempo todo el conocimiento se suma y le resultará menos costoso adquirirlo a través de la investigación, que por su propia experiencia.

Por otro lado la capacidad de adaptarse a los nuevos requerimientos y exigencias del mercado, es una habilidad con la que no todas las compañias cuentan hoy en dia. Es por esta razon que la planeacion estrategica toma gran importancia, ya que dicha herramienta facilita a la organizaciones el entendimiento y la capacidad de generar estrategias en pro del cumplimiento de objetivos y metas, para finalmente lograr el éxito organizacional.

Finalmente, la relevancia que ha tomado las diferentes maneras de gestionar las organizaciones conjunto todas las estrategias y herramientas existentes, se ha ido actualizando a medida que la competitividad entre las mismas va aumentando, todo con el fin de obtener una ventaja competitiva que logre generar un valor agregado para la compañía y que finalmente pueda posicionarla como una organizacion reconocida a nivel nacional e internacional.

\section{REFERENCIAS}

(1) Alvarez Torres, M. G. (2006). Manual de planeación estratégica : la metodología de consultoría más práctica para crecer en un ambiente competitivo. Panorama editorial.

(2) Ávila Cifuentes, O., Félix Antonio, C. A., \& Andrés Yesid, R. A. (2008). UNbizplanner: herramienta de software para preparar planes de negocios. Ingeniería e Investigación, 28(1), $154-161$.

(3) Blanco Gracía, S., \& Segovia Vargas, M. de J. (2014). Praxis del cálculo financiero. Madrid. 
(4) Botello peñaloza, H. A. (2016). Las certificaciones de calidad y la internacionalización de las firmas industriales colombianas. Suma de Negocios, 7(2), 73-81. [En línea] Disponible en: http://doi.org/10.1016/j.sumneg.2016.02.009

(5) Calvo Vérgez, J. (2011). La experiencia de los presupuestos participativos en los entes locales. Madrid.

(6) Cárdenas, M., \& Rozo, S. (2009). Informalidad empresarial en Colombia: problemas y soluciones. Desarrollo y Sociedad, (63), 211-243.

(7) Escandon-Barbosa, D. M., \& Hurtado-Ayala, A. (2016). Influencia de los estilos de liderazgo en el desempeño de las empresas exportadoras colombianas. Estudios Gerenciales, 32(139), 137-145. [En línea] Disponible en: http://doi.org/10.1016/j.estger.2016.04.001

(8) Fernandez Hurtado, S. R., \& Martinez Martinez, L. A. (2017). Internationalization of Colombian Firms: Competitive and Productivity as a Problem to Enter the Asian Market. Asian Social Science, 14(1), 183-192. [En línea] Disponible en: http://doi.org/10.5539/ass. v14n1p183

(9) Fernandez, S. R. (2013). It Takes Two to Tango: Commercial Relation Beyond Of Bilateral Agreement, China and Colombia to Sign a Free Trade Agreement. American Journal of Business and Management, 2(4), 275-295. [En línea] Disponible en: http://doi. org/10.11634/216796061302426

(10) González-Campo, C. H., \& Hurtado Ayala, A. (2014). Influencia de la capacidad de absorción sobre la innovación: un análisis empírico en las mipymes colombianas. Estudios Gerenciales, 30(132), 277-286. [En línea] Disponible en: http://doi.org/10.1016/j.estger.2014.02.015

(11) González Ramírez, M. de los R. (2001). Sistemas de información para la empresa. Universidad de Alicante.

(12) Hernández y Rodriguez, S. J. (2011). Introducción a la administración (Quinta). México.

(13) Hurtado, R. F. (2013). Initiative Analysis to Enter into Potential and Restricted Market : Small , Medium and Largest Enterprises Having Chance into a Virgin Market : Latin American ( CELAC ) and China ( Practical, China Case ). International Journal of Business and Management, 8(3), 103-122. [En línea] Disponible en: http://doi.org/10.5539/ijbm.v8n3p103

(14) Lerma González, H. D. (2009). Metodología de la investigación : propuesta, anteproyecto y proyecto (Vol. Cuarta edi). Bogotá, D.C.: Ecoe ediciones.

(15) Martín Armario, J., Ángeles Rastrollo Horrillo, M., \& González Robles, E. M. (2009). La internacionalización de la empresa: El conocimiento experimental como derterminante del resultado en mercados exteriores. Cuadernos de Economía y Dirección de La Empresa, 12(39), 123-149. [En línea] Disponible en: http://doi.org/10.1016/S1138-5758(09)70037-4 
(16) Martínez Carazo, P. C. (2010). Pyme. Estrategia para su internacionalización.: EBSCOhost (Primera). Barranquilla, Colombia: Ecoe Ediciones-Ediciones Uninorte.

(17) Martinez Martinez, L. A., \& Fernandez Hurtado, S. R. (2018). Internal Communication Issues in the Firms: Does It Affect the Productivity? Review of European Studies, 10(2), 1-13. [En línea] Disponible en: http://doi.org/10.5539/res.v10n2p1

(18) Mejía Argueta, C., Agudelo, I., \& Soto Cardona, O. C. (2016). Planeación por escenarios: un caso de estudio en una empresa de consultoría logística en Colombia. Estudios Gerenciales, 32(138), 96-107. [En línea] Disponible en: http://doi.org/10.1016/j.estger.2015.12.004

(19) Mendoza, J. M. (2012). Decisiones estratégicas : macroadministración. Barranquilla: Universidad del Norte.

(20) Meza Orozco, J. de J. (2010). Evaluación financiera de proyectos.

(21) Mintzberg, H. (1994, January). The Fall and Rise of Strategic Planning.

(22) Ochoa Díaz, H., \& Ríos Millán, A. M. (2011). Liderazgo, la competencia esencial que transformó una empresa colombiana en un gigante internacional: el caso de cementos Argos. Estudios Gerenciales, 27(121), 39-58.

(23) Porter, M. E. (1990). La ventaja competitiva de las naciones.

(24) Prieto Herrera, J. E. (2009). Investigación de mercados. Bogotá: Ecoe ediciones. [En línea] Disponible en: http://doi.org/9789586486200

(25) Rozas Gutiérrez, S., Corredor Velandia, C., Lombana Coy, J., Silva Guerra, H., Castellanos Ramírez, A., González Ortíz, J., \& O. (2013). Negocios internacionales : fundamentos y estrategias: EBSCOhost. : : 2 Edition, Revised Aumen. Barranquilla, Col : Universidad Del Norte, 404. [En línea] Disponible en: http://doi.org/9789587413564

(26) S. Rick, F. (2014). Empirical Result on Firms' Cluster Integration: Should Firms Evolve beyond Their Region? International Journal of Trade, Economics and Finance, 5(3), 204 211. [En línea] Disponible en: http://doi.org/10.7763/IJTEF.2014.V5.372

(27) Sánchez, J. C. (2012). Los métodos de investigación. Ediciones Díaz de Santos.

(28) Torres, M. G. A. (2006). Manual De Planeacion Estrategica/ Manual of Strategic Planning. Panorama Editorial. 